\title{
Upper extremity deep venous thrombosis prevalence in the NHS Grampian Medical Ambulatory clinic: diagnostic, therapeutic, and prognostic considerations in oncology patients
}

\author{
Stavroula Lila Kastora' ${ }^{1} \cdot$ Olusegun Oduyoye $^{1,2} \cdot$ Shafaq Mahmood ${ }^{2}$
}

Received: 25 June 2021 / Accepted: 2 September 2021 / Published online: 13 September 2021

(c) The Author(s) 2021

\begin{abstract}
Introduction Whilst upper extremity deep vein thromboses (UEDVT) account for approximately 5 to $10 \%$ of all cases of DVT, rigorous guidelines regarding diagnosis and management of presenting patients remain to be developed. The association of UEDVT with concurrent asymptomatic pulmonary embolism as well as the first presentation of malignancy deems essential rigorous research and clinical guideline development to ensure optimal patient care.

Methods This retrospective audit study is the first to provide estimates of UEDVT prevalence in the North-East Deanery main hospital centre, Aberdeen Royal Infirmary (ARI).

Results Of the 605 patients attending the ARI Ambulatory Emergency Care (AEC) clinic with clinical suspicion of UEDVT, 38 (6.2\%) had a confirmatory diagnosis. Underlying malignancy, presence of PICC line, and cardiovascular co-morbidities were identified as common confounding factors. Subclavian vein with concurrent extension to primarily the cephalic vein thrombosis was identified as the most commonly thrombosed venous territories. Importantly, oncology patients were found to have poorer survival outcomes following an UEDVT, in comparison to patients with other significant co-morbidities (cardiovascular, chronic renal disease, inflammatory bowel disease): HR 5.814 (95\%CI 1.15, 29.25), $p$ 0.012. Lastly, genetic associations were drawn between patient genetic status as tested for other co-morbidities and prothrombotic cellular cascades, suggesting rigorous VTE assessment in patients identified with congenital or acquired mutations, namely, in $C A L R, J A K$, $M S H 2 / 6, M Y C$, and FXN.

Conclusions Overall, this study offers the first report of UEDVT presentations in the UK with no restrictions of patient performance status or underlying co-morbidities and provides a rounded clinical picture of patient characteristics, diagnosis, management, and prognostic associations in view of rigorous guideline development.
\end{abstract}

Keywords Bioinformatics · Oncology $\cdot$ Prevalence $\cdot$ Survival analysis $\cdot$ Upper extremity deep venous thrombosis

\section{Introduction}

Primary upper extremity deep vein thrombosis (UEDVT) presents a rare clinical entity with an estimated annual incidence of 1 to 2 cases per 100,000 populations. Primary UEDVT, also referred to as Paget-Schroetter syndrome or "effort" thrombosis, is defined as thrombosis of the deep

Stavroula Lila Kastora

Stavroula.kastora@nhs.scot; Stavroula.kastora@abdn.ac.uk

1 Medical Sciences \& Nutrition, University of Aberdeen School of Medicine, Aberdeen, UK

2 Emergency Care Centre, Aberdeen Royal Infirmary, Foresterhill Campus, Aberdeen AB25 2ZN, UK veins draining the upper extremity due to anatomic abnormalities resulting in axillosubclavian compression [1, 2]. This clinical entity typically affects young and physically active individuals presenting as sudden, severe upper extremity pain, and swelling after strenuous upper-body activity. An aggressive therapeutic approach that comprises anticoagulation, catheter-directed thrombolysis, and thoracic outlet decompression present one of the fundamental treatment strategies at relieving acute symptoms and minimizing complications, such as recurrent thromboembolism and post-thrombotic syndrome, in this patient group. On the contrary, secondary or provoked UEDVT presents the majority of upper extremity thrombotic clinical presentations. Secondary UEDVTs primarily occur due to deep vein interventions including central or peripherally inserted catheters 
(PICC) and pacemaker or defibrillator insertions. Other provoking factors such as prothrombotic states such as inherited or acquired thrombophilia and malignancy have also been identified [3, 4]. Pulmonary embolism (PE) as a secondary thrombotic event following an UEDVT remains a serious complication and accounts for approximately $6 \%$ of cases [5] whilst the incidence of asymptomatic PE in patients with UEDVT may range from 3 to $36 \%$ [6].

The gold standard of UEDVT management as per causative factor remains obscure, potentially reflecting the rarity of UEDVT as a clinical presentation. For patients with clinically suspected UEDVT, Constans et al. (2008) combined clinical signs and symptoms with risk factors for UEDVT to derive a clinical decision score comparable with those used for lower-extremity DVT [7]. The feasibility and safety of a diagnostic algorithm for UEDVT were evaluated in the ARMOUR study, which was a multicentre prospective study on 406 patients with clinically suspected UEDVT [8].

Furthermore, guidelines on initial diagnosis or treatment options tailored to patient groups and their requirements are still to be developed. The timeline of maintenance of peripherally inserted central catheters after a thrombotic event also remains to be clarified (NICE guidelines) [9]. Lastly, whilst UEDVT has been investigated as a marker of occult malignancy in previous work, the clinical utility of searching for occult cancer in patients presenting with UEDVT remains unclear [10].

The aim of this retrospective audit study was to assess the prevalence of UEDVT in patients visiting the AEC Ambulatory Emergency Clinic (AEC) of the Aberdeen Royal Infirmary between 2015 and 2020, identify causative factors, and highlight commonalities in medical and interventional management tailored to patient presentation. Furthermore, we explored the potential of UEDVT to act as a prognostic factor of mortality in patients with underlying malignancy and putative genetic associations in this patient group.

Overall, this study offers the first report of upper extremity DVT presentations with no restrictions on patient performance status or underlying co-morbidities. Consequently, this study provides a rounded clinical picture of patient characteristics, diagnosis, management, and prognostic associations in view of rigorous guideline development.

\section{Materials and methods}

\section{Study design}

This is a retrospective audit study. Eligible for inclusion were all patients arriving in the Ambulatory Emergency Care (AEC) clinic with potential UEDVT during 2015-2020 in the Aberdeen Royal Infirmary (NHS Grampian). These patients were identified through filtering the AEC records according to presenting complaint.

\section{Data collection and definition of study exposure}

The following clinical and demographic data were collected from electronic clinical records: date of AEC presentation, age, gender, identification of deceased patients (date of death), BMI, co-morbidities (myocardial, respiratory, congenital and/or haematological conditions, malignancy), PICC or Pacemaker line insertion, date of insertion and date of removal, D-Dimer (requested-yes/no, result), imaging modality (ultrasound (US), Computerised Tomography (CT) Angiogram, Venogram), date of imaging modality, venous territories involved, treatment option (medical, interventional), and, if applicable duration, pharmacological treatment, and category of therapeutic agent. Electronic medical records were also studied for information on the UEDVT causative factors, genetic factors involved, type of therapeutic agent used (active agent), duration of use, recurrent UEDVT presentation, and specialty input according to patient confounding condition. Ethical approval was sought from the Research and Development department of the University of Aberdeen. Given that this study was conducted in the context of an audit, we were advised to register the study under the Quality assessment and improvement records of NHS Grampian database; Project code 5295. All patient identifiers were removed, and numerical series were used to code for each patient.

\section{Primary and secondary outcomes}

Among the primary outcomes of the study was to identify how many patients with potential UL-DVTs presenting in AEC were diagnosed with UEDVT. Another primary outcome involved the identification of primary vs. secondary UEDVTs, causative factors, and demographics. Secondary outcomes involved assessment of compliance with current guidelines regarding UL-DVT diagnosis (D-Dimer and imaging modality selection). UEDVT diagnosis is in oncology vs. patients with other co-morbidities and correlation with worsening survival prognosis.

\section{Statistical analysis}

The coded dataset is available upon request. Statistical analysis was performed using GraphPad Prism (v. 8). Pearson correlation analysis ( $R$-values) and statistical significance ( $p$-values) are available upon request. GraphPad (GP) statistical significance scale was employed to obtain four decimal significance values. Pearson correlation matrix was employed to compute the correlation coefficient for each pair of variables, regardless of other 
variables. Data were assumed to have been sampled from Gaussian distribution for continuous parameters; thus, the Pearson correlation test was employed. Survival analysis was conducted by Log-rank (Mantel-Cox) and GehanBreslow-Wilcoxon test.

STRING analysis of protein association as shown in Fig. 5, was performed with STRING: Functional Protein Association Networks (https://string-db.org) online platform. The addition of connective nodes was performed up to the first degree. Data were retrieved from text mining, experiments, and databases. Edge colours represented the protein-protein interactions as identified experimentally (pink, light blue) or predicted in silico (green-gene neighbourhood, red-gene fusions, blue-gene co-occurrence). Yellow edges indicate associations as identified via text mining whilst black edges signify co-expression.

\section{Results}

\section{Study participants}

A total of 605 patients were identified via the AEC records as potentially eligible for inclusion. The highest arrival peak was identified in 2018 [ $N$ 197]. The confirmed UEDVT diagnoses per year ranged from 3 in 2020 (min) to 10 in $2016(\max )($ mean 6.16; SD 2.48) (Fig. S1).

\section{Patient demographics and clinical details}

A total of 38 patients had a confirmed UEDVT, 6.2\% of total UEDVT potential presentations $(N 605)$. Other diagnoses included cellulitis (67\%; $N 405)$, trauma (24\%, $N 145)$, lymphoedema from known radiotherapy $(3 \%, 17)$. The median age at presentation was 57 , and mean BMI was 29.08 (Fig. 1A, B). A total of $50 \%$ of the patients ( $N$ 19) were male (Fig. 1C). In our patient cohort, $11.11 \%$ were smokers, and $6.11 \%$ were ex-smokers (Fig. 1D). The majority of patients had a significant underlying comorbidity. Namely, $24.73 \%$ had an active malignancy, out of which $23.68 \%$ required a PICC line insertion (Fig. 2A). Another significant risk factor was attributed to cardiovascular co-morbidities (including cardiomyopathy, previous myocardial infarction, hypertension, and familial hypercholesterolemia). A total of $21.05 \%$ [ $N$ 8] were diagnosed with one of the aforementioned cardiovascular illnesses, of which $5.26 \%\left[\begin{array}{ll}N & 2]\end{array}\right.$ required a pacemaker or defibrillator insertion (Fig. 2A). Only in 7.89\% [N 3] of the patients, active lifestyle, and increased physical activity (e.g., Paget-Schroetter) were identified as a provoking factor of UEDVT (Fig. 2A).
A.

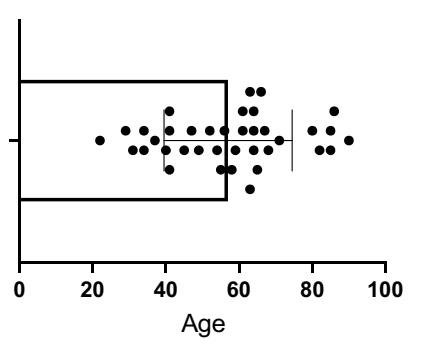

B.

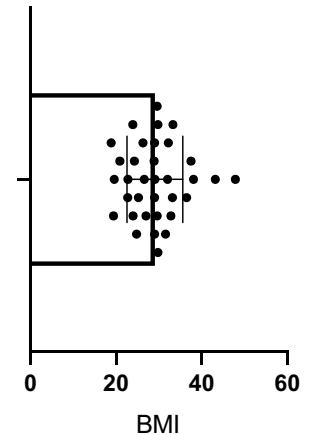

C.

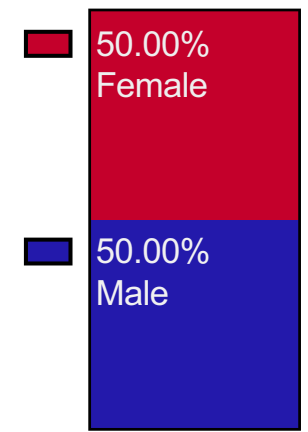

D.

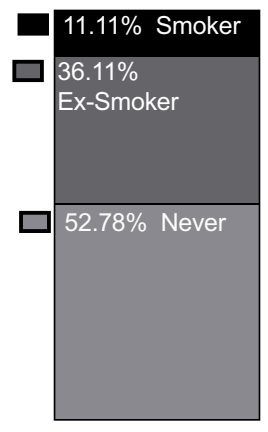

Fig. 1 Patient demographics (N38). A Age with median and SD. B BMI with mean and SD. C Gender. D Smoking status. The figure was generated using GraphPad Prism V. 8. BMI (body mass index), SD (standard deviation)

\section{Diagnostic modalities}

In our patient cohort, arm involvement was in $29.73 \%$ of the presentations right and $70.27 \%$ left arm (Fig. 3A).

A.

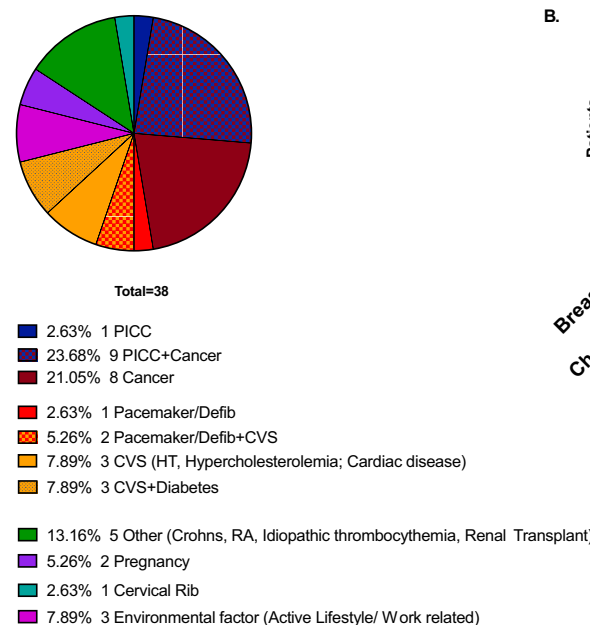

Fig. 2 Patient comorbidities. Pie chart of risk factors as identified in our cohort for developing UEDVT $\mathbf{A}$ and bar chart representation of malignancy type distribution in selected patient cohort B. The figure was generated using GraphPad Prism V. 8. *Peripherally inserted central catheter (PICC), T2DM (type 2 diabetes mellitus), Defib (defibrillator), CVS (Cardiovascular co-morbidities), HT (hypertension), RA (rheumatoid arthritis) 
Ultrasound was the imaging modality of choice according to national and international guidelines with $81.40 \%$ of the patients being diagnosed solely by ultrasound (Fig. 3B). Only 3 patients were primarily assessed with venogram, among which one required a further $\mathrm{CT}$ angiogram (Fig. 3B). This patient was diagnosed with cervical rib resulting in venous malformation. In almost for the entirety of our dataset (97.37\%), a D-Dimer was not requested, in compliance with the NICE guidelines (Fig. 3C) [9]. Analysis of venous territory involvement as per radiological report indicated that the subclavian was the primarily affected deep vein $(37.7 \%)$, followed by the axillary $(18.03 \%)$ and the basilic (13.11\%) vein (Fig. 3D). Subclavian thrombosis with concurrent extension to other venous territories occurred in $81.81 \%$ of the cases (18 out of 22) (Fig. 3D). Among the extended territories, cephalic vein thrombosis comprised $55.56 \%$ of the concurrent cases (Fig. 3D).

Pearson correlation analysis highlighted that the presence of thrombosis in any one of the venous territories often signifies further extension to other; the presence of cervical rib associates with DVTs in all upper extremity venous territories whilst PICC line insertion statistically correlates this basilic thrombotic events (Fig. S2). Patients with comorbidities, other than malignancy, were mostly treated with DOAC (direct oral anticoagulant) anticoagulation (44.73\%) (Fig. 4). Oncology patients or pregnant females were managed with LMWH (39.47\%). Two patients who had recurrent DVT (on previous apixaban; rivaroxaban) stepped up to warfarin and apixaban, respectively. Both had further co-morbidities (CVS, T2DM, PICC line insertion). The overall treatment period was 3 months regardless of pharmaceutical agent. Interventional management (thrombolysis, embolectomy) was reserved for only

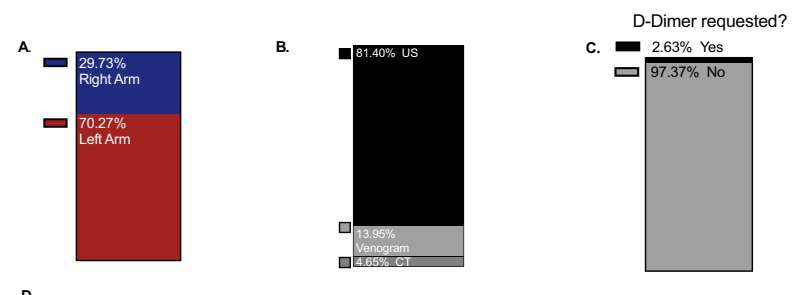

D.

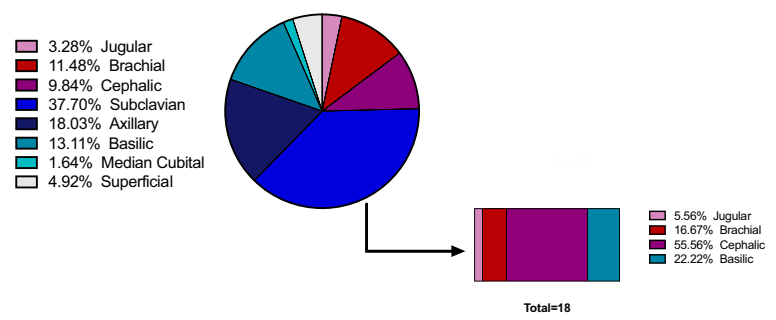

Fig. 3 DVT characteristics and incidence $(N=39)$. A Arm affected. B Treatment. C Diagnostic imaging modality. D Vein affected and subgroup analysis of subclavian DVTs extending to further vein territories. The figure was generated using GraphPad Prism V. 8. US (ultrasound), CT (computed tomography angiogram)
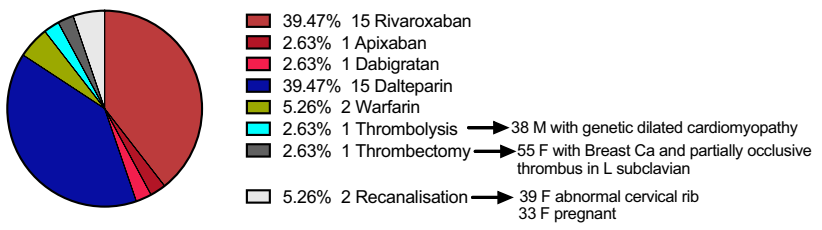

Fig. 4 UEDVT medical and interventional management (the figure was generated using GraphPad Prism V. 8. Female (F), breast cancer (Breast $\mathrm{Ca})$, left (L)

four patients (10.52\%), who, in their majority, were significantly younger than the cohort mean (Fig. 4).

\section{UEDVT as a prognostic factor in oncology patients}

Given that $27.03 \%$ of the patients with UEDVT were deceased at the time of the data collection, we explored the potential of UEDVT to act as a poor prognostic factor in specific disease mortality. Curve comparison probability of survival after UEDVT in patients with solid malignancy (breast: 6; colorectal 8; cholangiocarcinoma: 1; lung cancer: 1; non-Hodgkins lymphoma: 1; Fig. 2B) comparison to patients with other co-morbidities (cardiovascular; T2/T1 DM; cervical rib, other) indicated a significant association between the date of UEDVT diagnosis and date of death in oncology patients. The majority of oncology patients $(6$ out of 17 patients) deceased within the same year or 1 year after the UEDVT diagnosis (Fig. 5). Kaplan-Mayer curves between the two populations significantly differed with $p$ value of 0.012: chi-square 6.310 and HR 5.814 [95\% CI 1.15, 29.25] (Fig. 5). Overall, this analysis suggests that the probability of mortality post-UEDVT diagnosis with any other comorbidity as a baseline vs. malignancy is significantly increased in oncology patients.

\section{Genetic considerations}

Lastly, we sought to understand whether the genetic background of our patients, as examined for their underlying

A.

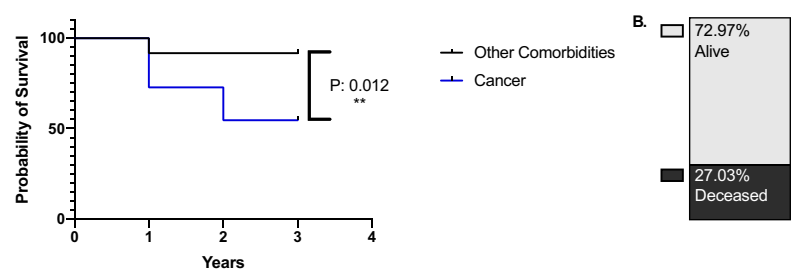

Fig. 5 Curve comparison probability of survival after upper extremity DVT in patients with malignancies (colorectal 6; HPB: 1; breast: 1 ) in comparison to patients with other co-morbidities (cardiovascular; type 2 diabetes mellitus; cervical rib, other). Chi-square 6.310; $p$-value $0.0120 ; \mathrm{MH} \log$ hazard ratio $\mathrm{A} / \mathrm{B}(0.03 ; 0.86)$. The figure was generated using GraphPad Prism V. 8 
co-morbidities, played a significant role in their prothrombotic baseline risk. A total of 5 out of the 38 patients had undergone genetic testing and counselling through the medical genetics department of the Aberdeen Royal infirmary. Conditions prompting genetic testing in this group included essential thrombocythemia (CALR; JAK), colorectal cancer ( $M S H 2 / 6, M Y C)$, Friedrich ataxia $(F X N)$, and long QT syndrome (SNTA1). The first-degree interactome of these genetic targets was identified through the ProteinProtein Interaction Networks Functional Enrichment Analysis database (STRING V. 11.0b) (Fig. 6A). A similar approach was employed to identify downstream interactome of factors assessed in routine thrombophilia screens (factor V Leiden, prothrombin, antithrombin, protein C, protein $\mathrm{S}$ ). Comparison of the first degree interactome of encoded proteins as identified in our patient cohort (PT list) and the routine thrombophilia screen (thrombophilia) assessed revealed an overlap of 52.8\% (N 19) of protein targets (Fig. 6B). This finding supports a solidified biological notion that we rarely see applied in clinical practice, that protein pathways are not restricted to particular phenotypes and pathway interplay is a common scenario. Consequently, whilst specific genetic mutations are identified in the context of explaining a clinical phenotype, it does not imply that they do not extend beyond that phenotype and affect other biological processes, specifically in our case prothrombotic cascades.

\section{Discussion}

In this work, we reviewed 605 patient files that were presented in the AEC, ARI with potential UEDVT between 2015 and 2020. We identified 38 patients with confirmed UEDVT.

Primary UEDVT remains a rare clinical entity and is mostly associated with strenuous upper extremity exercise.
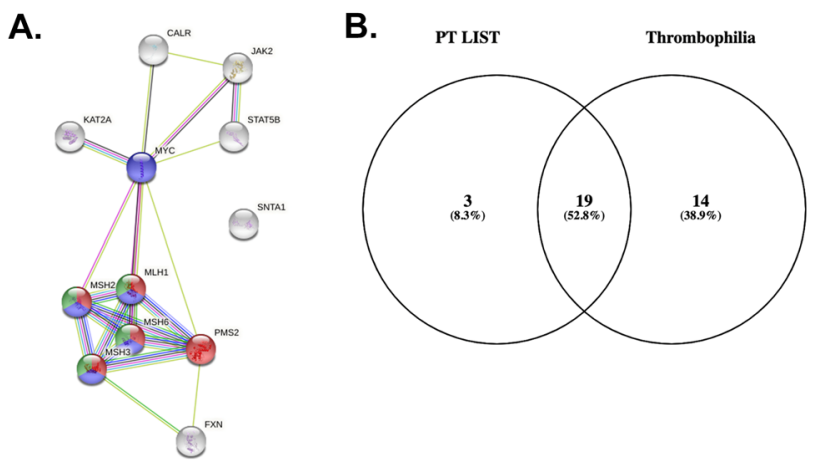

Fig. 6 First degree interactome of genetic targets as identified in our patient list $\mathbf{A}$ and overlap with the first degree interactome routinely screened protein players of thrombophilia screen B. PT list (genes identified in patients)
Conversely, secondary UEDVT remains a significant complication especially in patients requiring PICCs, with an incidence similar, if not greater than centrally inserted catheters, ranging from 3 to $58 \%$ [11]. The incidence of thrombosis in the upper extremity is significantly pronounced in the subclavian vein $(18-67 \%)$, followed by axillary (5-25\%) and the brachial (4-11\%) [12]. Concerning PICC-associated DVTs, marked predilection for the left side is observed [12]. This finding potentially reflects that the majority of patients are right-handed, and thus left-sided insertion, in the non-dominant hand is preferred. Among the main complications of UL-DVT, unlike lower limb thromboses, it remains increased mortality, recurrent thromboembolism, and post-thrombotic syndrome. The mortality rate in this patient group can range from 10 to $50 \%$, related mainly to the underlying malignancy, and fatal pulmonary embolism which may contribute to the overall mortality [13]. Our findings, in agreement with recent literature highlighted that use of PICC line, preexisting cardiovascular conditions, and malignancy, represent independent risk factors of UEDVT [14].

Regarding occult malignancy, UEDVT occurring in the absence of increased activity or thrombophilia and with no identifiable anatomic abnormalities or other contributing benign risk factors should raise concerns of occult malignancy. Up to $25 \%$ of patients will be diagnosed with malignancy, within 1 year of UEDVT [15]. Regarding patients with already diagnosed malignancies, UEDVT has been hypothesised to be a poor prognostic factor and may signify increased mortality, a finding that remains to be confirmed. Another clinical consideration for UEDVT presenting patients should be the investigation of an underlying thrombophilic state, and screening for thrombophilia should be included in the initial evaluation [16]. Intriguingly, the overall prevalence of hypercoagulable states in patients with thrombosis of the upper extremities was not significantly higher than in control subjects but was significantly lower than that in patients with deep leg vein thrombosis $[17,18]$.

In terms of diagnosis, D-Dimer is rarely informative and is not recommended as per NICE guidelines. Consequently, the suggested diagnostic modality is upper extremity Doppler ultrasound, whilst it should be mentioned that contrast venography is more standardised, given the lack of operator subjectivity, and sensitive. A systematic review of 17 studies concluded that compression ultrasonography is an acceptable alternative to standard contrast venography [19]. The summary estimates of the sensitivity of compression, Doppler ultrasound, and Doppler ultrasound with compression were 97,84 , and $81 \%$, respectively, with specificity 96,94 , and $93 \%$, respectively. Of note, regardless of the efficiency of US in UEDVT diagnosis, disadvantages include 
technician-dependent error and that mural or proximal subclavian thrombi may not be adequately visualised due to acoustic shadowing by the overlying structures $[19,20]$.

Given the rarity of UEDVT, regional and national guidelines regarding work-up and management of affected patients remain obscure. This study aimed to identify the prevalence of diagnosed UEDVTs and confounding risk factors in an ambulatory clinical setting, that serves a large proportion of the rural population in North East Scotland. We further aimed to assess compliance within the limits of current guidelines regarding UEDVT diagnosis (D-Dimer and imaging modality and treatment). We also present a potential flow chart of consistent medical assessment and management of patients with potential UEDVT to ensure cost-effective but, importantly, thorough patient work-up, minimizing misdiagnosis or suboptimal management (Fig. 7).

\section{Strengths and limitations}

Whilst this work is limited by the inherent limitations of a retrospective audit report and may significantly benefit by a broadened base of patients, including medical and surgical, important findings have been raised that warrant investigation in a larger cohort setting. We highlighted that an UEDVT diagnosis in oncology patients signifies worsening survival outcomes when compared to patients with other comorbidities. This finding comes in support with previous hypotheses [12, 14, 15]. Importantly, we also highlighted, that genetic mutations identified in our patient cohort within other disease investigation frameworks, may affect other biological processes, specifically prothrombotic cascades, explaining UEDVT presentations. This result warrants further research to identify whether this is an incidental finding, or it should be incorporated in the baseline clinical considerations regarding medical genetic testing for congenital conditions.

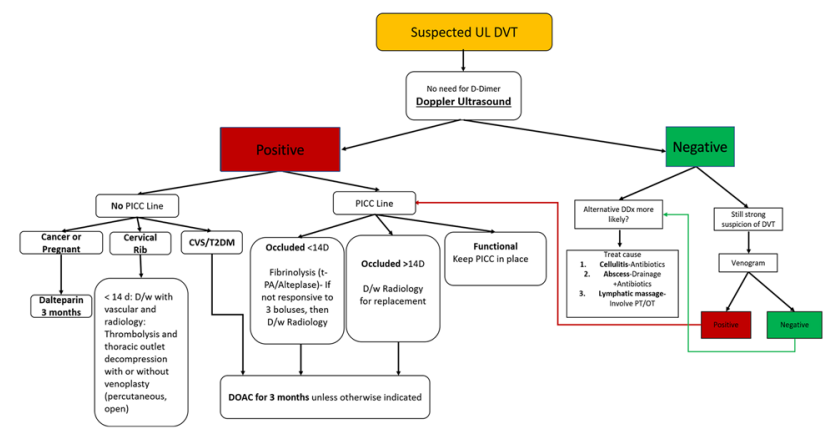

Fig. 7 Flow chart of UEDVT patient assessment and downstream medical or surgical management. Peripherally inserted central catheter (PICC), T2DM (type 2 diabetes mellitus), Defib (defibrillator), CVS (cardiovascular co-morbidities), direct oral anticoagulant (DOAC)

\section{Conclusion}

There is no "one size fits all" approach to the treatment of UEDVT. Tailored care is required given the plethora of underlying factors that may contribute to the presentation, with special consideration to the potential of underlying malignancy. Guidelines regarding the clinical approach and management of the first UEDVT presentation remain to be developed.

Supplementary Information The online version contains supplementary material available at https://doi.org/10.1007/s11845-021-02775-0.

Author contribution Search and data extraction: SLK, SM. Data statistical analysis: SLK. Original draft preparation: SLK. Conceptualisation: OO. Expert opinion: OO. Final manuscript and approval: SLK, OO, SM.

\section{Declarations}

Statement of ethics This study was conducted in the context of an audit; the project was registered under the quality assessment and improvement records of NHS Grampian database; Project code 5295.

Conflict of interest The authors declare no competing interests.

Open Access This article is licensed under a Creative Commons Attribution 4.0 International License, which permits use, sharing, adaptation, distribution and reproduction in any medium or format, as long as you give appropriate credit to the original author(s) and the source, provide a link to the Creative Commons licence, and indicate if changes were made. The images or other third party material in this article are included in the article's Creative Commons licence, unless indicated otherwise in a credit line to the material. If material is not included in the article's Creative Commons licence and your intended use is not permitted by statutory regulation or exceeds the permitted use, you will need to obtain permission directly from the copyright holder. To view a copy of this licence, visit http://creativecommons.org/licenses/by/4.0/.

\section{References}

1. Isma N, Svensson PJ, Gottsäter A, Lindblad B (2010) Upper extremity deep venous thrombosis in the population-based Malmö thrombophilia study (MATS). Epidemiology, risk factors, recurrence risk, and mortality. Thrombosis research 125(6):e335-8

2. Illig KA, Doyle AJ (2010) A comprehensive review of PagetSchroetter syndrome. J Vasc Surg 51(6):1538-47

3. Kucher N (2011) Mar 3 Deep-vein thrombosis of the upper extremities. N Engl J Med 364(9):861-869

4. Lindblad B, Tengborn L, Bergqvist D (1988) Deep vein thrombosis of the axillary-subclavian veins: epidemiologic data, effects of different types of treatment and late sequelae. Eur J Vasc Surg 2(3):161-5

5. Muñoz FJ, Mismetti P, Poggio R et al (2008) Clinical outcome of patients with upper-extremity deep vein thrombosis: results from the RIETE Registry. Chest 133(1):143-8

6. Heil J, Miesbach W, Vogl T et al (2017) Deep vein thrombosis of the upper extremity: a systematic review. Dtsch Arztebl Int 114(14):244 
7. Constans J, Salmi LR, Sevestre-Pietri MA et al (2008) A clinical prediction score for upper extremity deep venous thrombosis. Thromb Haemost 99(01):202-207

8. Kleinjan A, Di Nisio M, Beyer-Westendorf J et al (2014) Safety and feasibility of a diagnostic algorithm combining clinical probability, d-dimer testing, and ultrasonography for suspected upper extremity deep venous thrombosis: a prospective management study. Ann Intern Med 160(7):451-457

9. National Institute for Health and Care Excellence (2021) Prevention and management of venous thromboembolism [NICE Guideline No. 122]. https://www.evidence.nhs.uk/document?id=2250587\& returnUrl=search $\% 3 \mathrm{fps} \% 3 \mathrm{~d} 100 \% 26 \mathrm{q} \% 3 \mathrm{ddvt} \% 252$ bguidelines

10. Adelborg K, Horváth-Puhó E, Sundbøll J et al (2018) Risk and prognosis of cancer after upper-extremity deep venous thrombosis: a population-based cohort study. Thromb Res 161:106-10

11. Chopra V, Anand S, Hickner A et al (2013) Risk of venous thromboembolism associated with peripherally inserted central catheters: a systematic review and meta-analysis. The Lancet 382(9889):311-25

12. Prescott SM, Tikoff GE (1979) Deep venous thrombosis of the upper extremity: a reappraisal. Circulation 59(2):350-5

13. Tohme S, Vancheswaran A, Mobbs K et al (2021) Predictable risk factors of upper-extremity deep venous thrombosis in a level $\mathrm{i}$ trauma center. International Journal of General Medicine 14:2637

14. Baarslag HJ, Koopman MM, Hutten BA et al (2004) Long-term follow-up of patients with suspected deep vein thrombosis of the upper extremity: survival, risk factors and post-thrombotic syndrome. Eur J Intern Med 15(8):503-7
15. Girolami A, Prandoni P, Zanon E et al (1999) Venous thromboses of upper extremities are more frequently associated with occult cancer as compared with those of lower limbs. Blood coagulation \& fibrinolysis: an international journal in haemostasis and thrombosis 10(8):455-7

16. Hendler MF, Meschengieser SS, Blanco AN et al (2004) Primary upper-extremity deep vein thrombosis: high prevalence of thrombophilic defects. Am J Hematol 76(4):330-7

17. Martinelli I, Cattaneo M, Panzeri D et al (1997) May 1 Risk factors for deep venous thrombosis of the upper extremities. Ann Intern Med 126(9):707-11

18. Di Nisio M, Van Sluis GL, Bossuyt PM et al (2010) Accuracy of diagnostic tests for clinically suspected upper extremity deep vein thrombosis: a systematic review. J Thromb Haemost 8(4):684-92

19. Mustafa BO, Rathbun SW, Whitsett TL, Raskob GE (2002) Sensitivity and specificity of ultrasonography in the diagnosis of upper extremity deep vein thrombosis: a systematic review. Arch Intern Med 162(4):401-4

20. Haire WD, Lynch TG, Lund GB et al (1991) Limitations of magnetic resonance imaging and ultrasound-directed (duplex) scanning in the diagnosis of subclavian vein thrombosis. J Vasc Surg 13(3):391-7

Publisher's Note Springer Nature remains neutral with regard to jurisdictional claims in published maps and institutional affiliations. 